\title{
Transjugular intrahepatic portosystemic shunt assisted with trans-splenic access
}

\author{
Zhang Cheng, Feng Wang, and Jianfei Liu \\ Department of Interventional Therapy, the First Affiliated Hospital of Dalian Medical University, Liaoning Province, China
}

Dear Editor,

Thrombosis of the portal vein, superior mesenteric vein, and splenic vein affects blood flow, which is caused by portal hypertension. Portal hypertension leads to the dilatation of collateral portosystemic veins along the gastrointestinal tract. The prevalence of nonneoplastic portal vein thrombosis (PVT) in liver cirrhosis ranges from $7.2 \%$ to $17 \%$, and is higher at the decompensated or advanced stage.' Transjugular intrahepatic portosystemic shunt (TIPS) placement should be considered as an option for such patients. Transsplenic access makes the procedure technically straightforward and should be considered as the primary method for recanalization. ${ }^{2}$

A 54-year-old male patient with recurrent periumbilical pain for 15 days was hospitalized. His vital signs were stable. The initial laboratory findings were as follows: hemoglobin $16.6 \mathrm{~g} / \mathrm{dL}$, platelet levels $270 \times 10^{9} / \mathrm{L}$, prothrombin time 11.9 seconds, international normalized ratio 1.05, fibrinogen $4.93 \mathrm{~g} / \mathrm{L}$, plasma D-dimer 1,970 $\mu \mathrm{g} / \mathrm{L}$, serum aspartate transaminase levels $69 \mathrm{U} / \mathrm{L}$, serum alanine transaminase levels $58 \mathrm{U} / \mathrm{L}$, serum total bilirubin levels $16.0 \mu \mathrm{mol} / \mathrm{L}$, serum albumin levels $6.9 \mu \mathrm{mol} / \mathrm{L}$. Perinuclear anti-neutrophil cytoplasmic antibodies (-), cytoplasmic anti-neutrophil cytoplasmic antibodies (-), anticardiolipin antibody immunoglobulin G/immunoglobulin M (-/-), and CA125 levels $41.52 \mathrm{U} / \mathrm{mL}$.
Gastric endoscopy revealed gastric bleeding, gastric ulcer (stage A1), and chronic non-atrophic gastritis (Fig. 1). Enhanced computed tomography of the upper abdomen showing massive filling defects in the portal vein, splenic vein, and superior mesenteric vein should be considered for thrombosis (Fig. 2). Portal hypertension is likely to lead to bleeding.

Percutaneous transhepatic portal venography was performed for the first time. Venography showed multiple filling defects in the right, left, and main portal vein suggesting PVT. The guidewire could not advance beyond the narrow segment of the main portal vein. Because the procedure using transhepatic access was not working, splenic vein was punctured under the guidance of ultrasound. After successful puncturing the splenic vein, splenic venography showed filling defects in the splenic and main portal vein with collateral vein formation around the stomach (Fig. 3A). The guide-wire catheter passed through the occlusion segment to the main portal vein. And then, the right internal jugular vein was punctured, and the RUPS-100 puncture system (Cook, Bloomington, IL, USA) was delivered through the jugular vein puncture route. TIPS was created between the right portal vein and the right hepatic vein. Balloon catheters of $4 / 80 \mathrm{~mm}, 6 / 60 \mathrm{~mm}$, and $8 / 60 \mathrm{~mm}$ (Evercross, ev3 Inc., Plymouth, MA, USA) were used to expand the main splenic vein and portal vein. Along the shunt

\section{Abbreviations:}

PVT, portal vein thrombosis; TIPS, transjugular intrahepatic portosystemic shunt

\section{Corresponding author : Feng Wang}

Department of Interventional Therapy, the First Affiliated Hospital of Dalian Medical University, 222 Zhongshan Rd, Xigang Qu, Dalian Shi, Liaoning Province 116011, China

Tel: +86-18042682287, Fax: +86-411-83622844

E-mail: 995953474@qq.com

https://orcid.org/0000-0001-6778-8240

Received : Mar. 15, 2019/ Revised : Apr. 17, 2019/ Accepted : Apr. 29, 2019 
way, a 10×60 mm covered stent (Fluency, Bard, Karisruhe, Germany) and a $10 \times 80 \mathrm{~mm}$ bare stent (Smart control, Cordis, Miami, $\mathrm{FL}$, USA) were placed to entirely cover the shunt. Angiography indicated satisfactory stent position, smooth passage of the contrast agent, and unobstructed blood flow through the splenic vein, main portal vein, and TIPS (Fig. 3B). Finally, the transsplenic access was closed with coils.

Risk factors associated with slow portal vein blood flow include age, gender, non-selective beta-blocker use, portosystemic shunting (e.g., spontaneous splenorenal shunt), severity of liver disease (as defined by Child-Turcotte-Pugh class), and splenectomy. ${ }^{3}$ PVT frequently occurs in patients with cirrhosis of the liver. It may also

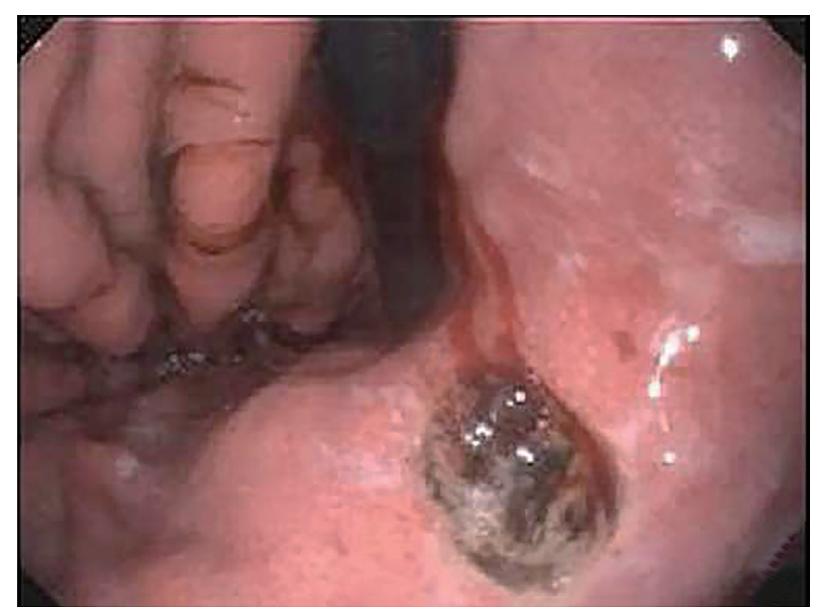

Figure 1. Endoscopy shows an approximately $1 \mathrm{~cm}$ sized deep ulcer with minor bleeding in the gastric cardia. Mucosa edema and hyperemia were observed in the gastric fundus and body.
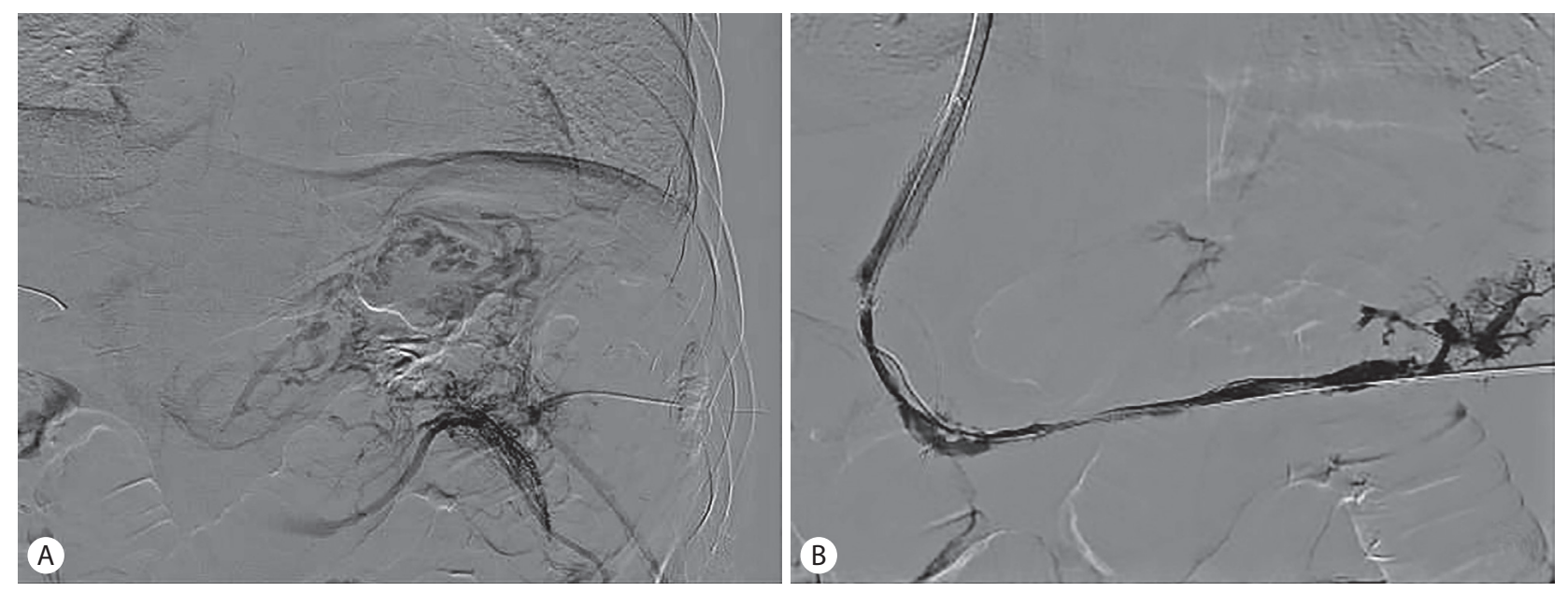

Figure 3. Intraoperative angiography. (A) Splenic venography (detector format size 30) shows thrombosis of splenic vein and portal vein. Note multiple collateral vein around the stomach. (B) Splenic venography after transjugular intrahepatic portosystemic shunt placement (detector format size 42) shows intact blood flow though the main portal vein. Note that multiple collateral veins around the stomach were dissapeared. 
traceptives, and malignancies with or without the above prothrombotic causes. The intra-abdominal inflammatory conditions leading to vascular endothelial injury can cause PVT. These include pancreatitis, ${ }^{5}$ cholangitis, appendicitis, and liver abscess. Local injury to the portal venous axis following splenectomy, laparoscopic colectomy, or abdominal trauma with the above acquired or inherited prothrombotic conditions can lead to PVT.

Splenoportography is now accepted as a safe procedure. ${ }^{6}$ As TIPS advances in its role as a therapeutic option in patients with PVT, it is important to consider the transsplenic approach given its high success rate and clinical effectiveness. ${ }^{7}$ In patients with PVT, traditional TIPS procedure may be difficult to confirm the portal vein puncture because blood regurgitation may be absent after the portal vein puncture. Transsplenic access may faciliitate the TIPS procedure because portal vein itself can be confirmed and guidewire in the portal vein can be used as a puncture target. In some reports, ${ }^{2,7}$ snare wire is placed within the thrombosed portal vein via trans-splenic access, and snare wire is used as a puncture target. Guidewire advanced through the needle can be caught by the snare and through-and-through technique can be applied between jugular vein access and splenic vein access.

\section{Author's contributions}

Zhang Cheng wrote the first version of manuscript. Feng Wang helped the first author to revise the process. Jianfei Liu helped to choose the photos. All authors participated in final approval of manuscript.

\section{Acknowledgements}

The editor of "Gut and Liver" found that our article is interesting and has potential, and he invited us to submit this article to Clinical and Molecular Hepatology, so we would like to thank about this.

\section{Conflicts of Interest}

The authors have no conflicts to disclose.

\section{REFERENCES}

1. Jiang TT, Luo XP, Sun JM, Gao J. Clinical outcomes of transcatheter selective superior mesenteric artery urokinase infusion therapy vs transjugular intrahepatic portosystemic shunt in patients with cirrhosis and acute portal vein thrombosis. World J Gastroenterol 2017;23:7470-7477.

2. Thornburg B, Desai K, Hickey R, Kulik L, Ganger D, Baker T, et al. Portal vein recanalization and transjugular intrahepatic portosystemic shunt creation for chronic portal vein thrombosis: technical considerations. Tech Vasc Interv Radiol 2016;19:52-60.

3. Stine JG, Northup PG. Management of non-tumoral portal vein thrombosis in patients with cirrhosis. Dig Dis Sci 2019;64:619-626.

4. Denninger MH, Chaït Y, Casadevall N, Hillaire S, Guillin MC, Bezeaud $\mathrm{A}$, et al. Cause of portal or hepatic venous thrombosis in adults: the role of multiple concurrent factors. Hepatology 2000;31:587591.

5. Ghelfi J, Thony F, Frandon J, Rodiere M, Leroy V, Vendrell A. Gastrointestinal bleeding due to pancreatitis-induced splenic vein thrombosis: treatment with percutaneous splenic vein recanalization. Diagn Interv Imaging 2016;97:677-679.

6. Chu HH, Kim HC, Jae HJ, Yi NJ, Lee KW, Suh KS, et al. Percutaneous transsplenic access to the portal vein for management of vascular complication in patients with chronic liver disease. Cardiovasc Intervent Radiol 2012;35:1388-1395.

7. Habib A, Desai K, Hickey R, Thornburg B, Vouche M, Vogelzang RL, et al. Portal vein recanalization-transjugularintrahepatic portosystemic shunt using the transsplenic approach to achieve transplant candidacy in patients with chronic portal vein thrombosis. J Vasc Interv Radiol 2015;26:499-506. 